\title{
Chromatic number of Harary graphs
}

\author{
Adel P. Kazemi* and Parvin Jalilolghadr \\ College of Mathematical Sciences, University of Mohaghegh Ardabili, P.O.Box 5619911367, Ardabil, Iran \\ * Corresponding author \\ E-mail: adelpkazemi@yahoo.com, a.p.kazemi@uma.ac.ir, p_jalilolghadr@yahoo.com
}

\begin{abstract}
A proper coloring of a graph $G$ is a function from the vertices of the graph to a set of colors such that any two adjacent vertices have different colors, and the chromatic number of $G$ is the minimum number of colors needed in a proper coloring of a graph. In this paper, we will find the chromatic number of the Harary graphs, which are the circulant graphs in some cases.
\end{abstract}

2010 Mathematics Subject Classification. 05C15.

Keywords. Harary graph, circulant graph, chromatic number.

\section{Introduction}

All graphs considered here are finite, undirected and simple. For standard graph theory terminology not given here we refer to [3]. Let $G=(V, E)$ be a graph with the vertex set $V$ of order $n$ and the edge set $E$ of size $m$.

A proper coloring of a graph $G$ is a function from the vertices of the graph to a set of colors such that any two adjacent vertices have different colors, and the chromatic number $\chi(G)$ of $G$ is the minimum number of colors needed in a proper coloring of a graph [3]. In a proper coloring of a graph a color class is the independent set of all same colored vertices of the graph. If $f$ is a proper coloring of $G$ with the color classes $V_{1}, V_{2}, \ldots, V_{l}$ such that every vertex in $V_{i}$ has color $i$, we simply write $f=\left(V_{1}, V_{2}, \ldots, V_{l}\right)$.

As you see in many references such as [3], the Harary graphs are defined as follows: given $2 m<n$, place $n$ vertices around a circle, equally spaced. Form $H_{2 m, n}$ by making each vertex adjacent to the nearest $m$ vertices in each direction around the circle. If $n$ is even, form $H_{2 m+1, n}$ by making each vertex adjacent to the nearest $m$ vertices in each direction and to the diametrically opposite vertex. Both kinds are regular. When $n$ is odd, index the vertices by the integers modulo $n$. Construct $H_{2 m+1, n}$ from $H_{2 m, n}$ by adding the edges $i \leftrightarrow i+\frac{n-1}{2}$ for $1 \leq i \leq \frac{n+1}{2}$. Obviously, $H_{2, n}=C_{n}$ and $H_{n-1, n}=K_{n}$ which $C_{n}$ and $K_{n}$ denote the cycle and complete graph of order $n$, respectively. These cases are not the subject of our study.

For $[n]=\{1,2, \ldots, n\}$ and a subset $D$ of it, the circulant graph $G(n, D)$ is a graph with the vertex set $[n]$, and $i j$ is an edge if and only if $i-j$ (to modulo $n$ ) belongs to $D \cup(-D)$. The study of chromatic number of circulant graphs of small degree has been widely considered in many papers such as $[1,2,4]$.

Every one can see that the Harary graph $H_{2 m, n}$ is the circulant graph $G(n, D)$ with $D=$ $\{1,2, \ldots, m\}$, and the Harary graph $H_{2 m+1, n}$ is the circulant graph $G(n, D)$ with $D=\{1,2, \ldots, m\} \cup$ $\{n / 2\}$ when $n$ is even, while for odd $n$ the Harary graph $H_{2 m+1, n}$ is not a circulant graph. Here, we will find the chromatic number of Harary graphs. 
Recall that for a graph $G$ of order $n, \alpha(G)$ or simply $\alpha$ denotes the independence number of $G$, which is the maximum cardinality of an independent set $S$ in $G$. It can be easily verify that for any Harary graph $H$,

$$
\alpha(H)= \begin{cases}\left\lfloor\frac{n}{m+1}\right\rfloor-1 & \text { if } H=H_{2 m+1, n}, n \text { is even and } n \equiv 0 \quad(\bmod 2 m+2), \\ \left\lfloor\frac{n}{m+1}\right\rfloor & \text { otherwise. }\end{cases}
$$

By considering this fact that each color class is an independent set, and every independent set has cardinality at most $\alpha(G)$, we conclude

$$
\chi(G) \geq\left\lceil\frac{n}{\alpha(G)}\right\rceil .
$$

Since the Harary graph $H_{m, n}$ is a complete graph of order $n$ if and only if $n=m+1$, and so its chromatic number is $n$, so in this paper, we always assume that $n>m+1$, and prove

$$
\chi\left(H_{m, n}\right)= \begin{cases}\left\lceil\frac{n}{\alpha}\right\rceil+1 & \text { if } n=m+3 \geq 10, \text { and } m \equiv 3 \quad(\bmod 4), \\ \left\lceil\frac{n}{\alpha}\right\rceil & \text { otherwise. }\end{cases}
$$

Through this paper, we consider

$$
n-t \equiv s \quad(\bmod t-1)
$$

where $t=\left\lceil\frac{n}{\alpha}\right\rceil$, and $\alpha$ denotes the independence number of a Harary graph.

\section{The chromatic number of $H_{2 m, n}$}

In the following theorem we prove $\chi\left(H_{2 m, n}\right)=\left\lceil\frac{n}{\alpha}\right\rceil$.

Theorem 2.1. For any integers $m>1$ and $n \geq 2 m+2, \chi\left(H_{2 m, n}\right)=\left\lceil\frac{n}{\alpha}\right\rceil$.

Proof. Since $\alpha=\left\lfloor\frac{n}{m+1}\right\rfloor$, we may assume that $n=\alpha(m+1)+r$, for some $0 \leq r \leq m$. Let $r=0$. Since for each $1 \leq i \leq m+1$, the set $V_{i}=\{i+(m+1) j \mid 0 \leq j \leq \alpha-1\}$ is independent, we conclude $\chi\left(H_{2 m, n}\right)=m+1=\left\lceil\frac{n}{\alpha}\right\rceil$, by (1.1). So, we may assume $r \neq 0$. Clearly, $t>m+1$, and we continue our proof in the following two cases.

Case 1: $n-m>(\alpha-1) t$. Let $f=\left(V_{1}, V_{2}, \ldots, V_{t}\right)$ be a coloring function of $H_{2 m, n}$ in which

$$
V_{i}=\{i+k t \mid 0 \leq k \leq \alpha-1\}, \quad \text { for } 1 \leq i \leq n-(\alpha-1) t,
$$

and

$$
V_{i}=\{i+k t \mid 0 \leq k \leq \alpha-2\}, \quad \text { for } n-(\alpha-1) t+1 \leq i \leq t .
$$

The condition $n-m>(\alpha-1) t$ guarantees that each of the sets $V_{i}$ is independent, and so $\chi\left(H_{2 m, n}\right)=$ $m+1=\left\lceil\frac{n}{\alpha}\right\rceil$, by $(1.1)$.

Case 2: $n-m \leq(\alpha-1) t$. Let $f=\left(V_{1}, V_{2}, \ldots, V_{t}\right)$ be a coloring function of $H_{2 m, n}$ in which

$$
V_{i}=\{i+k t \mid 0 \leq k \leq s-1\} \cup\{i+s t+k(t-1) \mid 0 \leq k \leq \alpha-s-1\},
$$


for $1 \leq i \leq t-1$, and $V_{t}=\{n\} \cup \mathcal{B}$, where

$$
\mathcal{B}= \begin{cases}\varnothing & \text { if } \quad s=0 \\ \{k t \mid 1 \leq k \leq s\} & \text { if } \quad s \neq 0\end{cases}
$$

Since $\left|V_{t}\right|=s+1$ and $\left|V_{i}\right|=\alpha$ for each $1 \leq i \leq t-1$, and $n=\alpha(t-1)+s+1$, we conclude that the distance between every two vertices in each $V_{i}$ is at least $t-1$, ans so $t-1 \geq m+1$ implies that each of the sets $V_{i}$ is independent. Hence $\chi\left(H_{2 m, n}\right)=m+1=\left\lceil\frac{n}{\alpha}\right\rceil$, by (1.1).

Q.E.D.

\section{The chromatic number of $H_{2 m+1, n}$ with even $n$}

By considering this fact that

$$
\alpha\left(H_{2 m+1, n}\right)= \begin{cases}\left\lfloor\frac{n}{m+1}\right\rfloor-1 & \text { if } n \text { is even and } n \equiv 0 \quad(\bmod 2 m+2), \\ \left\lfloor\frac{n}{m+1}\right\rfloor & \text { otherwise, }\end{cases}
$$

we have $n=\alpha(m+1)+r$ or $n=(\alpha+1)(m+1)+r$, for some $0 \leq r \leq m$. Now, let $r=0$. In the first case, $\chi\left(H_{2 m+1, n}\right)=m+1=t$ and in the second case, $t>m+1$. If $r \neq 0$, then $2 m+2 \nmid n$ and so $n=\alpha(m+1)+r$ for some $1 \leq r \leq m$ which easily implies that $t>m+1$. So in this section, Without loss of generality, we may assume that $t>m+1$. Also, without loss of generality, we may assume that $s \neq 0$ if $n-m \leq(\alpha-1) t$. Because $s=0$ implies $2 t-2 \nmid n$, and so the set $\{1+k(t-1) \mid 0 \leq k \leq \alpha-s-1\}$ is independent for each $1 \leq i \leq t$. Therefore $\chi\left(H_{2 m+1, n}\right)=t$.

To find the chromatic number of the Harary graph $H_{2 m+1, n}$, with even $n$, we need the following two lemmas.

Lemma 3.1. Let $n-m \leq(\alpha-1) t$. If $2 t \mid n$, then $\frac{n}{2 t}>s$.

Proof. Set $\frac{n}{2 t}:=q$. First, let $\frac{n}{2}-s t=j(t-1)-i t$, for some $0 \leq i \leq s-1$ and some $1 \leq j \leq \alpha-s-1$. Then $(i+q-s) t=j(t-1)$. Since $t$ and $t-1$ are coprime, $j$ is a multiple of $t$ and $i+q-s$ is a multiple of $t-1$. Specially, $i+q-s \geq t-1$. Moreover, $i-s \leq-1$ and so $t-1 \leq i+q-s \leq q-1$ which implies that $t \leq q$. Since $q \leq s$ implies $t \leq s$, we have $q>s$.

Now, let $\frac{n}{2}-s t \neq j(t-1)-i t$, for each $0 \leq i \leq s-1$ and each $1 \leq j \leq \alpha-s-1$. By knowing $n=\alpha(t-1)+s+1$, since $q \leq s$ implies $\frac{n}{2}-s t=j(t-1)-i t$ for $i=q-1$ and $j=\alpha-s-1$, we obtain $q>s$.

Q.E.D.

Let $V_{i}^{\prime}=\{i+k t \mid 0 \leq k \leq s-1\}$ and $V_{i}^{\prime \prime}=\{i+s t+k(t-1) \mid 0 \leq k \leq \alpha-s-1\}$, for $1 \leq i \leq t$, be subsets of the vertex set of the Harary graph $H_{2 m+1, n}$. We note that $V_{i}^{\prime} \cap V_{i}^{\prime \prime}=\varnothing$, and $V_{i}^{\prime}$ is independent, by Lemma 3.1, because $V_{i}^{\prime}$ is independent if and only if either $2 t \nmid n$ or $2 t \mid n$ and $\frac{n}{2 t}>s$. Also $V_{i}^{\prime \prime}$ is independent if and only if either $2 t-2 \nmid n$ or $2 t-2 \mid n$ and $\frac{n}{2 t-2}>\alpha-s-1$. So for each $0 \leq i \leq s-1$, the set $V_{i}=V_{i}^{\prime} \cup V_{i}^{\prime \prime}$ is independent if and only if the set $V_{i}^{\prime \prime}$ is independent and $\frac{n}{2}-s t \neq j(t-1)-i t$, for each $1 \leq j \leq \alpha-s-1$. Next lemma states that if $\frac{n}{2}-s t=j(t-1)-i t$ for some $0 \leq i \leq s-1$ and some $1 \leq j \leq \alpha-s-1$, then the set $V_{i}^{\prime \prime}$ is again independent.

Lemma 3.2. Let $n-m \leq(\alpha-1) t$. If $\frac{n}{2}-s t=k(t-1)-p t$ for some $0 \leq p \leq s-1$ and some $1 \leq k \leq \alpha-s-1$, then the set $V_{i}^{\prime \prime}=\{i+s t+k(t-1) \mid 0 \leq k \leq \alpha-s-1\}$ is independent, where $1 \leq i \leq t$ 
Proof. It is sufficient to prove $2 t-2 \nmid n$. Let $2 t-2 \mid n$ and $\frac{n}{2}-s t=k(t-1)-p t$, for some $0 \leq p \leq s-1$ and some $1 \leq k \leq \alpha-s-1$. Then $(s-p) t=\left(\frac{n}{2 t-2}-k\right)(t-1)$. Since $t$ and $t-1$ are coprime, it follows that $\frac{n}{2 t-2}-k$ is a multiple of $t$ and $s-p$ is a multiple of $t-1$, specially $t-1 \leq s-p$. On the other hand, we have $s+1 \leq t-1$, which implies $p \leq-1$, a contradiction. Hence $2 t-2 \nmid n$, and so the set $V_{i}^{\prime \prime}$ is independent, where $1 \leq i \leq t$.

Q.E.D.

Theorem 3.3. For each even $n$ with $n \geq 2 m+3$,

$$
\chi\left(H_{2 m+1, n}\right)= \begin{cases}\left\lceil\frac{n}{\alpha}\right\rceil+1 & \text { if } n=2 m+4 \geq 10, \text { and } m \equiv 1 \quad(\bmod 2), \\ \left\lceil\frac{n}{\alpha}\right\rceil & \text { otherwise }\end{cases}
$$

Proof. First let $n=2 m+4 \geq 10$ and $m \equiv 1(\bmod 2)$. Let $\chi\left(H_{2 m+1, n}\right)=t=\left\lceil\frac{n}{\alpha}\right\rceil$, and let $f$ be a proper coloring function of $H_{2 m+1} n$. Since the subgraph of the graph induced by the vertices 1,2 , $\ldots, m+1$ is a clique, we may assume that $f(i)=i$ for each $1 \leq i \leq m+1$. Then for each vertex $m+2 \leq i \leq 2 m+4$,

$$
\begin{aligned}
& f(m+2) \in\{1, m+2\}, f(m+3) \in\{2, m+2\}, f(n) \in\{m+1, m+2\}, \\
& f(n-1) \in\{m, m+2\}, f(m+i) \in\{i-3, i-1, m+2\}, 4 \leq i \leq m+2 .
\end{aligned}
$$

Now we discuss on the following two cases.

Case 1. $f(m+2)=m+2$. Then $f(n)=m+1$, and $f(m+2 i+1)=2 i$, for $1 \leq i \leq \frac{m+1}{2}$, which implies $f(n)=f(n-2)=m+1$, a contradiction. Because the distance between the vertices $n$ and $n-2$ is less than $m$.

Case 2. $f(m+2)=1$. First, by a proof similar to Case 1 , we have $f(n) \neq m+2$. Hence $f(n)=m+1$. We also see that for at most one vertex $m+3 \leq i \leq n-1$, we may have $f(i)=m+2$. Let $f(m+3)=m+2$. Then $f(n-1)=m$, which implies $f(m+2 i)=2 i-1$, for $2 \leq i \leq \frac{m+1}{2}$, a contradiction. Hence $f(m+3)=2$. Also, with a similar proof, we will have $f(n-1) \neq m+2$. If for each vertex $m+3 \leq i \leq n-1, f(i) \neq m+2$, then $f(n-2 i-1)=m-2 i$, for $0 \leq i \leq \frac{m-1}{2}$, a contradiction (Because $f(m+4)=f(m+2)=1$ ). Therefore, for only one vertex $m+3 \leq i \leq n-2, f(i)=m+2$. Then $f(n-1)=m$ and $f(n-3) \in\{m+2, m-2\}$.

Since $f(n-3)=m+2$ implies $f(n-2)=m+1$, which is a contradiction to $f(n)=m+1$, we have $f(n-3)=m-2$. Then $f(n-5) \in\{m+2, m-4\}$. By continuing this method, we will have $f(m+4)=m+2$. Hence $f(n+2 i+1)=2 i$, for $2 \leq i \leq \frac{m+1}{2}$, a contradiction. Therefore $\chi\left(H_{2 m+1, n}\right)>\left\lceil\frac{n}{\alpha}\right\rceil$. Now since the coloring function $f$ with criterion

$$
\begin{gathered}
f(i)=i, \text { for } 1 \leq i \leq m+2, f(m+3)=2, f(m+4)=3, f(m+5)=m+3, \\
f(m+2 i)=2 i-1, f(m+2 i+1)=2 i-2, \text { for } 3 \leq i \leq \frac{m+3}{2}
\end{gathered}
$$

is a proper coloring of the graph with $\left\lceil\frac{n}{\alpha}\right\rceil+1$ colors, we obtain $\chi\left(H_{2 m+1, n}\right)=\left\lceil\frac{n}{\alpha}\right\rceil+1$, where $n=2 m+4$ and $m$ is odd.

Now, in the second part of our proof, we may assume that if $n=2 m+4 \geq 10$, then $m \equiv 0$ $(\bmod 2)$, and we continue our proof in the following four cases. We recall that $t=\left\lceil\frac{n}{\alpha}\right\rceil$. 
Case 1. $n-m>(\alpha-1) t$ and $2 t \nmid n$. Let $f=\left(V_{1}, V_{2}, \ldots, V_{t}\right)$ be a coloring function of $H_{2 m+1, n}$ in which

$$
V_{i}=\{i+k t \mid 0 \leq k \leq \alpha-1\}, \quad \text { for } 1 \leq i \leq n-(\alpha-1) t,
$$

and

$$
V_{i}=\{i+k t \mid 0 \leq k \leq \alpha-2\}, \quad \text { for } n-(\alpha-1) t+1 \leq i \leq t .
$$

Since the given coloring function $f=\left(V_{1}, V_{2}, \ldots, V_{t}\right)$ is a proper coloring of $H_{2 m+1, n}$, we obtain $\chi\left(H_{2 m+1, n}\right)=\left\lceil\frac{n}{\alpha}\right\rceil$.

Case 2. $n-m>(\alpha-1) t$ and $2 t \mid n$. For even $t$, let

$$
\begin{gathered}
V_{2 i-1}=\{2 i+k t \mid 0 \leq k \leq n / 2 t-1\} \cup\{2 i-1+k t \mid n / 2 t \leq k \leq \alpha-1\}, \\
V_{2 i}=\{2 i-1+k t \mid 0 \leq k \leq n / 2 t-1\} \cup\{2 i+k t \mid n / 2 t \leq k \leq \alpha-1\},
\end{gathered}
$$

where $1 \leq i \leq t / 2$, and for odd $t$, let

$$
\begin{gathered}
V_{2 i-1}=\{2 i+k t \mid 0 \leq k \leq n / 2 t-1\} \cup\{2 i-1+k t \mid n / 2 t \leq k \leq \alpha-1\}, \\
V_{2 i}=\{2 i-1+k t \mid 0 \leq k \leq n / 2 t-1\} \cup\{2 i+k t \mid n / 2 t \leq k \leq \alpha-1\},
\end{gathered}
$$

where $1 \leq i \leq(t-3) / 2$, and

$$
\begin{gathered}
V_{t-2}=\{k t-1 \mid 1 \leq k \leq n / 2 t\} \cup\{k t-2 \mid n / 2 t+1 \leq k \leq \alpha-1\} \cup\{n\}, \\
V_{t-1}=\{k t \mid 1 \leq k \leq n / 2 t\} \cup\{k t-1 \mid n / 2 t+1 \leq k \leq \alpha-1\} \cup\{n-2\}, \\
V_{t}=\{k t-2 \mid 1 \leq k \leq n / 2 t\} \cup\{k t \mid n / 2 t+1 \leq k \leq \alpha-1\} \cup\{n-1\} .
\end{gathered}
$$

In each case, the given coloring function $f=\left(V_{1}, V_{2}, \ldots, V_{t}\right)$ is a proper coloring of $H_{2 m+1, n}$, and so $\chi\left(H_{2 m+1, n}\right)=\left\lceil\frac{n}{\alpha}\right\rceil$.

Case 3. $n-m \leq(\alpha-1) t$ and $\frac{n}{2}-s t \neq j(t-1)-i t$, for each $0 \leq i \leq s-1$ and each $1 \leq j \leq \alpha-s-1$. Let $V_{i}^{\prime}=\{i+k t \mid 0 \leq k \leq s-1\}$ and $V_{i}^{\prime \prime}=\{i+s t+k(t-1) \mid 0 \leq k \leq \alpha-s-1\}$ be subsets of the vertex set of the Harary graph $H_{2 m+1, n}$, where $1 \leq i \leq t$. We note that $V_{i}^{\prime} \cap V_{i}^{\prime \prime}=\varnothing$, and $V_{i}^{\prime}$ is independent, by Lemma 3.2.

First let either $2 t-2 \nmid n$ or $2 t-2 \mid n$ and $\frac{n}{2 t-2}>\alpha-s-1$. Then the set $V_{i}^{\prime \prime}$ is independent, and this condition that $\frac{n}{2}-s t \neq j(t-1)-i t$ for each $0 \leq i \leq s-1$ and each $1 \leq j \leq \alpha-s-1$, implies that each of the sets $V_{i}=V_{i}^{\prime} \cup V_{i}^{\prime \prime}$ is independent. Therefore the coloring function $f=\left(V_{1}, V_{2}, \ldots, V_{t}\right)$ is a proper coloring of $H_{2 m+1, n}$, where

$$
V_{i}=\{i+k t \mid 0 \leq k \leq s-1\} \cup\{i+s t+k(t-1) \mid 0 \leq k \leq \alpha-s-1\},
$$

for $1 \leq i \leq t-1$, and $V_{t}=\{k t \mid 1 \leq k \leq s\} \cup\{n\}$. Hence $\chi\left(H_{2 m+1, n}\right)=\left\lceil\frac{n}{\alpha}\right\rceil$.

Now, let $2 t-2 \mid n$ and $\frac{n}{2 t-2} \leq \alpha-s-1$. Then the given coloring function $f=\left(V_{1}, V_{2}, \ldots, V_{t}\right)$ is a proper coloring of $H_{2 m+1, n}$, where

$$
\begin{aligned}
V_{i} & =\{i+k t \mid 0 \leq k \leq s\} \\
& \cup\{i+s t+k(t-1) \mid 1 \leq k \leq n /(2 t-2)-1\} \\
& \cup \quad\{i+1+s t+k(t-1) \mid n /(2 t-2) \leq k \leq \alpha-s-1\}
\end{aligned}
$$


for each $1 \leq i \leq t-1$, and $V_{t}=\{k t \mid 1 \leq k \leq s\} \cup\left\{1+s t+\frac{n}{2}\right\}$. Hence $\chi\left(H_{2 m+1, n}\right)=\left\lceil\frac{n}{\alpha}\right\rceil$.

Case 4. $n-m \leq(\alpha-1) t$ and $\frac{n}{2}-s t=q(t-1)-p t$, for some $0 \leq p \leq s-1$ and some $1 \leq q \leq \alpha-s-1$. Then each of the sets $V_{i}^{\prime \prime}=\{i+s t+k(t-1) \mid 0 \leq k \leq \alpha-s-1\}$ is independent, by Lemma 3.2. Now we define for odd $t$,

$$
\begin{aligned}
V_{2 l-1}= & \{2 l-1+k t \mid 0 \leq k \leq p-1\} \\
\cup & \{2 l+p t\} \\
\cup & \{2 l-1+k t \mid p+1 \leq k \leq s-1\} \\
\cup & V_{2 l-1}^{\prime \prime}, \\
V_{2 l} \quad & \{2 l+k t \mid 0 \leq k \leq p-1\} \\
& \cup\{2 l-1+p t\} \\
\cup & \{2 l+k t \mid p+1 \leq k \leq s-1\} \\
& \cup V_{2 l}^{\prime \prime}, \\
& V_{t}=\{k t \mid 1 \leq k \leq s\} \cup\{n\},
\end{aligned}
$$

while for even $t$,

$$
\begin{aligned}
V_{2 l-1}= & \{2 l-1+k t \mid 0 \leq k \leq p-1\} \\
\cup & \{2 l+p t\} \\
\cup & \{2 l-1+k t \mid p+1 \leq k \leq s-1\} \\
\cup & V_{2 l-1}^{\prime \prime}, \\
V_{2 l}= & \{2 l+k t \mid 0 \leq k \leq p-1\} \\
& \cup \quad\{2 l-1+p t\} \\
\cup & \{2 l+k t \mid p+1 \leq k \leq s-1\} \\
& \cup V_{2 l}^{\prime \prime}
\end{aligned}
$$

where $1 \leq l \leq\left\lfloor\frac{t}{2}\right\rfloor$. Then the coloring function $f=\left(V_{1}, V_{2}, \ldots, V_{t}\right)$ is a proper coloring of $H_{2 m+1, n}$, and so $\chi\left(H_{2 m+1, n}\right)=\left\lceil\frac{n}{\alpha}\right\rceil$.

\section{The chromatic number of $H_{2 m+1, n}$ with odd $n$}

Since $\alpha\left(H_{2 m+1, n}\right)=\left\lfloor\frac{n}{m+1}\right\rfloor$ for odd $n$, we have $n=\alpha(m+1)+r$ for some $0 \leq r \leq m$. Without loss of generality, we may assume that $t>m+1$. A simple calculation shows that if $s=0$, then $\chi\left(H_{2 m+1, n}\right)=\left\lceil\frac{n}{\alpha}\right\rceil$ (we recall that $n-t \equiv s(\bmod t-1)$, and $\left.t=\left\lceil\frac{n}{\alpha}\right\rceil\right)$. So we assume $s \neq 0$.

Theorem 4.1. For each odd $n$ with $n \geq 2 m+3, \chi\left(H_{2 m+1, n}\right)=\left\lceil\frac{n}{\alpha}\right\rceil$.

Proof. We presend our proof in the following two cases.

Case 1. $n-m>(\alpha-1) t$. We first prove that $2 t \nmid n-1$. Assume on the contrary $2 t \mid n-1$. Then $n=1+s+\alpha(t-1)$ implies that $\alpha-s=\left(\alpha-\frac{n-1}{t}\right) t$. The condition $\alpha \neq s$ implies that $\alpha-s \geq t$ and so $t<\alpha$. On the other hand, since $n=\alpha(m+1)+r$ for some $0 \leq r \leq m$, we obtain

$$
\begin{aligned}
n-m & \leq \alpha(m+1) \\
\leq & \alpha(t-1) \\
& <(\alpha-1) t,
\end{aligned}
$$


which is a contradiction. Therefore $2 t \nmid n-1$. Now let $f=\left(V_{1}, V_{2}, \ldots, V_{t}\right)$ be a coloring function of $H_{2 m+1, n}$, in which

$$
V_{i}=\{i+k t \mid 0 \leq k \leq \alpha-1\}, \quad \text { where } 1 \leq i \leq n-(\alpha-1) t,
$$

and

$$
V_{i}=\{i+k t \mid 0 \leq k \leq \alpha-2\}, \quad \text { where } n-(\alpha-1) t+1 \leq i \leq t .
$$

Since the condition $n-m>(\alpha-1) t$ guarantees that each of the sets $V_{i}$ is independent, we obtain $\chi\left(H_{2 m+1, n}\right)=\left\lceil\frac{n}{\alpha}\right\rceil$.

Case 2. $n-m \leq(\alpha-1) t$. For $1 \leq i \leq t$, let $V_{i}^{\prime}=\{i+k t \mid 0 \leq k \leq s-1\}$ and $V_{i}^{\prime \prime}=$ $\{i+s t+k(t-1) \mid 0 \leq k \leq \alpha-s-1\}$ be subsets of the vertex set of the Harary graph $H_{2 m+1, n}$. Since $V_{i}^{\prime}$ is independent if and only if either $2 t \nmid n-1$ or $2 t \mid n-1$ and $\frac{n-1}{2 t}>s$, so to prove that $V_{i}^{\prime}$ is independent, it is sufficient to show that if $2 t \mid n-1$, then $\frac{n-1}{2 t}>s$. Since $n=1+s t+(\alpha-s)(t-1)$, we obtain $\left(\frac{n-1}{t}-s\right) t=(\alpha-s)(t-1)$. Then $\frac{n-1}{t}-s$ is a multiple of $t-1$ and $\alpha-s$ is a multiple of $t$. In particular, $\frac{n-1}{t}-s \geq t-1$. Since by the definition of $s, s<t-1$, we obtain $\frac{n-1}{2 t}>s$.

Since also $V_{i}^{\prime \prime}$ is independent if and only if either $2 t-2 \nmid n-1$ or $2 t-2 \mid n-1$ and $\frac{n-1}{2 t-2}>\alpha-s-1$, to prove that $V_{i}^{\prime \prime}$ is independent, it is sufficient to show that $2 t-2 \nmid n-1$. For this aim, let $n-t=k(t-1)+s$ for some integers $k$ and $0<s \leq t-2$. Then $n-1=(k+1)(t-1)+s$ implies $t-1 \nmid n-1$, and so $2 t-2 \nmid n-1$.

Therefore $V_{i}=V_{i}^{\prime} \cup V_{i}^{\prime \prime}$ is independent if and only if $\frac{n-1}{2}-s t \neq j(t-1)-i t$, for every $0 \leq i \leq s-1$ and every $1 \leq j \leq \alpha-s-1$. So, without loss of generality, we may assume that $\frac{n-1}{2}-s t=q(t-1)-p t$ for some integers $0 \leq p \leq s-1$ and $1 \leq q \leq \alpha-s-1$. Now we define for odd $t$,

$$
\begin{aligned}
V_{2 l-1}= & \{2 l-1+k t \mid 0 \leq k \leq p-1\} \\
\cup & \{2 l+p t\} \\
\cup & \{2 l-1+k t \mid p+1 \leq k \leq s-1\} \\
\cup & V_{2 l-1}^{\prime \prime}, \\
V_{2 l}= & \{2 l+k t \mid 0 \leq k \leq p-1\} \\
\cup & \{2 l-1+p t\} \\
\cup & \{2 l+k t \mid p+1 \leq k \leq s-1\} \\
& \cup V_{2 l}^{\prime \prime}, \\
& V_{t}=\{k t \mid 1 \leq k \leq s\} \cup\{n\},
\end{aligned}
$$

where $1 \leq l \leq\left\lfloor\frac{t}{2}\right\rfloor$, while for even $t$,

$$
\begin{aligned}
V_{2 l-1} & =\{2 l-1+k t \mid 0 \leq k \leq p-1\} \\
& \cup\{2 l+p t\} \\
& \cup\{2 l-1+k t \mid p+1 \leq k \leq s-1\} \\
& \cup \quad V_{2 l-1}^{\prime \prime} \\
V_{2 l} & =\{2 l+k t \mid 0 \leq k \leq p-1\} \\
& \cup\{2 l-1+p t\} \\
& \cup\{2 l+k t \mid p+1 \leq k \leq s-1\} \\
& \cup \quad V_{2 l}^{\prime \prime}
\end{aligned}
$$


where $1 \leq l \leq\left\lfloor\frac{t}{2}\right\rfloor$. Then the coloring function $f=\left(V_{1}, V_{2}, \ldots, V_{t}\right)$ is a proper coloring of $H_{2 m+1, n}$, and so $\chi\left(H_{2 m+1, n}\right)=\left\lceil\frac{n}{\alpha}\right\rceil$.

Q.E.D.

\section{References}

[1] J. Barajas and O. Serra, On the chromatic number of circulant graphs, Discrete Math, 309 (2009), 5687-5696.

[2] C. Heuberger, On planarity and colorability of circulant graphs, Discrete Math, 268 (2003), 153-169.

[3] D. B. West, Introduction to Graph Theory, 2nd ed, prentice hall, USA, (2001).

[4] H. G. Yeh and X. Zhu, 4-colourable 6-regular toroidal graphs, Discrete Math. 273 (2003), 261274. 